\title{
Network complexity and species traits mediate the effects of biological invasions on dynamic food webs
}

\author{
Miguel Lurgi ${ }^{1,2}$, Núria Galiana ${ }^{1}$, Bernat C. López ${ }^{1,3}$, Lucas N. Joppa ${ }^{4}$ and José M. Montoya ${ }^{1,2 *}$ \\ ${ }^{1}$ CREAF, Cerdanyola del Vallès, Spain \\ ${ }^{2}$ Departamento de Biología Marina y Oceanografía, Instituto de Ciencias del Mar, Agencia Consejo Superior de Investigaciones Científicas, Barcelona, Spain \\ ${ }^{3}$ Unitat d'Ecologia, Departament de Biologia Animal, Biologia Vegetal i Ecologia, Universitat Autònoma de Barcelona, Cerdanyola del Vallès, Spain \\ ${ }^{4}$ Microsoft Research, Cambridge, UK
}

\section{Edited by:}

Stefano Allesina, University of

Chicago, USA

\section{Reviewed by:}

Matías Arim, Universidad de la República, Uruguay

Jacopo Grilli, Università degli Studi

di Padova, Italy

*Correspondence:

José M. Montoya, Departamento de Biología Marina y Oceanografía, Instituto de Ciencias del Mar,

Passeig Marítim de la Barceloneta

37-49, 08003 Barcelona, Spain

e-mail:montoya@icm.csic.es
Biological invasions are a major threat to natural communities worldwide. While several species traits have been identified as important determinants of invasion success, a systematic exploration of the effects of invasions on native communities, and the role of species and community features on community robustness in the face of invasion is lacking. We present a theoretical approximation considering food web structure and species population dynamics to study the effects of invasions on complex food webs. We find that less complex (i.e., less connected) food webs are more resistant to invasions. Simulated invasions promote profound changes in several food web properties and stability measures, such as decreases in modularity and the number of food chains from basal to top species; and a decoupling of community- and population-level temporal variability. Additionally, species traits such as body size and diet breadth are strong determinants of invasion success across several trophic levels, with larger and more generalist species being more successful invaders in general. Our work complements species-centered invasion studies by adding a more holistic and systematic perspective to the study of invasions on species interaction networks.

Keywords: dynamical systems, ecological networks, network complexity, stability, invasive species, community assembly, bio-energetic model, species interactions

\section{INTRODUCTION}

Biological invasions are one of the main threats to biodiversity worldwide with far reaching consequences for ecological communities and local economies (Vitousek et al., 1996; Cardinale et al., 2006). Ever since Elton's seminal work (1958), ecologists have sought to understand biological invasions on natural communities. Two main strains of research have derived from these efforts: (i) understanding the effects of invasions on native communities (e.g., Vitousek et al., 1996; Simberloff et al., 2013), and (ii) identifying common features-from both native communities and invasive species-that allow predictions of invasion success (e.g., Kolar and Lodge, 2002; Shea and Chesson, 2002). Research focusing on the first of these topics has revealed that invasions usually decrease native biodiversity and reduce population abundances (Kenis et al., 2008; Vilà et al., 2011), and affect ecosystem functioning (Wardle et al., 2011; Simberloff et al., 2013). The impacts of invasive species on native communities are however, heterogeneous and not unidirectional, making general predictions of species response and effects too system-dependent and idiosyncratic (Vilà et al., 2011). Although several studies have considered the relationship between community and food web structure and biological invasions (e.g., Elton, 1958; Case, 1990; Pimm, 1991; Romanuk et al., 2009), at present, we lack a more general and predictive framework for understanding the effects of species introductions in complex networks of interacting species. On the other hand, efforts directed toward the latter point have identified several species traits as successful predictors of invasion success. These traits vary across the taxonomic group of the invader under consideration (Williamson and Fitter, 1996; Kolar and Lodge, 2002; Cassey et al., 2004). As a consequence, some researchers have claimed that there are no rules governing the invasion process with any real predictive value (Bright, 1998) because invasion success is strongly species or taxonomic group-dependent. Including species traits into theoretical frameworks for understanding and predicting the impact of invasive species on native communities has thus been identified as a key aspect to advance the study of biological invasions (Ricciardi et al., 2013). The characteristics of the invaded community also mediate invasion success. Experimental studies have shown the importance of the structure of multitrophic communities for the establishment of invaders: zooplankton diversity, for example, provides biotic resistance against invasion by algae (Dzialowski et al., 2006). However, the difficulties associated with collecting empirical data from large species interaction networks call for theoretical approximations to address this issue. Several authors have suggested comprehensive theoretical frameworks for the study of invasions in a community context (Shea and Chesson, 2002; van Kleunen et al., 2010; Blackburn et al., 2011).

The role of food web structure in determining invasion success has long been recognized in the theoretical literature. Early theoretical competitive community assembly experiments, with simple Lotka-Volterra dynamics, showed that more connected 
(Pimm, 1991) and strongly interacting (Case, 1990) communities were more resistant to invasion. These studies however, used small and randomly assembled communities, and only addressed which community features made them more robust against invasion. Theoretical explorations within larger communities displaying more realistic food web structures are needed to determine both the role of food web complexity and species traits on invasion success. Recently, Romanuk et al. (2009) explored a number of food web and invader properties that predisposed species to successfully invade model communities. Using more realistic food web configurations and population dynamics determined by a bio-energetic model, they found that more complex communities were more robust to invasions, with complexity measured as network connectance (i.e., the fraction of realized links in the web). The trophic position and generality (i.e., number of prey species) of the invader were the best predictors of success.

Here we integrate food web structure and non-linear population dynamics in large, complex model communities. In particular, we (i) identify key features of food webs (in addition to species richness and connectance) that make them more robust to invasions, (ii) investigate the effects of invasion on several community properties, and (iii) explore traits that can determine species invasion success including body size and prey capturing efficiency. We test the following hypotheses: (a) more complex communities will be more robust to invasions, (b) successful invasions will alter food web structure and dynamics by changing their composition and complexity, which will in turn make native communities more susceptible to further perturbations, (c) species with larger body sizes, more abundant, and more able to capture prey will be more successful invaders, and (d) generalist consumers and prey attacked by less predators are likewise more capable of invading communities. Our study complements recent efforts toward the understanding of the effects of biological invasions on real complex food webs (Galiana et al., 2014) by providing a framework on which a wider range of food web structures, species traits, and stability mechanisms can be systematically evaluated.

\section{MATERIALS AND METHODS FOOD WEB GENERATION}

Food web structure of native communities was obtained using the niche model (Williams and Martinez, 2000). This model generates food webs by simply using the number of species $(S)$ and connectance $\left(C=L / S^{2}\right)$ of the network as input parameters. It is based on an algorithm that arranges species in a one-dimensional "niche" and assigns predator and prey links to each species in the network in a hierarchical way.

We performed in silico invasion experiments on food webs containing 60 species $(S=60)$ and 3 different values of connectance $(C=0.05,0.1,0.15)$ that fall within the range observed in empirical food webs (Dunne et al., 2002; Montoya and Solé, 2003). This allowed us to have relatively species-rich communities with different levels of complexity-defined here by $C$. Previous studies looking at several aspects of community dynamics and stability using a similar bioenergetic model, have considered smaller communities, up to 40 species (Brose et al., 2006; Binzer et al., 2011) or 30 species in the case of Romanuk et al. (2009).

\section{NON-LINEAR MODEL FOR POPULATION DYNAMICS}

For simulating species population dynamics we used an allometric version of the bio-energetic model originally proposed by Yodzis and Innes (1992), which was updated with new allometric coefficients and extended to multispecies systems by Williams and Martinez (2004). In this model, the change in species biomass through time is given by:

$$
\begin{aligned}
& \dot{B}_{i}=r_{i} G_{i} B_{i}-\sum_{j} \frac{x_{j} y B_{j} F_{j i}}{e_{j i}} \\
& \dot{B}_{i}=-x_{i} B_{i}+\sum_{k} x_{i} y B_{i} F_{i k}-\sum_{j} \frac{x_{j} y B_{j} F_{j i}}{e_{j i}}
\end{aligned}
$$

Equations (1) and (2) give the rate of change in biomass through time for producers and consumers in the food web, respectively. $B_{i}$, and $B_{j}$ denote the biomass density of populations $i$ and $j, r_{i}$ is the mass-specific intrinsic growth rate of producer species $i$, and $G_{i}$ is the logistic growth function. $x_{i}$ and $x_{j}$ denote the mass-specific metabolic rates of species $i$ and $j, y$ the consumer's maximum consumption rate, which in the present study takes the same value for all species in the system (see Supplementary Material S2), and $e_{j i}$ is the assimilation efficiency of consumer $j$ when consuming prey $i$. The subscript $k$ denotes the resources of $i$, and the subscript $j$ its consumers. $F_{j i}$ corresponds to the functional response (detailed in Equation 4).

The term for the logistic growth is given by:

$$
G_{i}=\left(1-\frac{B_{i}}{K_{i}}\right)
$$

which assumes that the growth of each basal species $i$ is dependent on its carrying capacity $K_{i}$ and that there is no direct competition among basal species. Thus, $G_{i}$ describes $i$ 's population biomass deviation from $K_{i}$. In the present formulation of the model all basal species have the same carrying capacity (see Supplementary S1 in Supporting Information for a detailed explanation).

In Equations (1) and (2), $F_{j i}$ corresponds to the functional response (i.e., the resource density-dependent feeding rate of consumer $j$ on a resource $i$ influenced by all the other resources $k$ of $j$ ), defined as:

$$
F_{j i}=\frac{\omega_{j i} B_{i}^{h}}{B_{0}^{h}+c B_{j}+\omega_{j i} B_{i}^{h}+\sum_{k} \omega_{j k} B_{k}^{h}}
$$

where $\omega_{j i}$ is a dimensionless positive weight factor sometimes used to simulate prey-preference, but that here is set to 0.5 for all interactions in order to ensure that predators do not exhaust their resources quickly without including prey preferences (see KoenAlonso, 2007 for details), $B_{0}$ is the half-saturation density, and $c$ and $h$ correspond to predator interference and Hill exponent terms, respectively. The Hill exponent determines the shape of the functional response: a large Hill exponent renders the curve more sigmoidal, which is interpreted as a refugee effect for the prey at low densities. 
Biological rates of production, metabolism, and maximum consumption are scaled allometrically following Binzer et al. (2011) (see Supplementary Materials S1, S2 in SI for details).

\section{FOOD WEB PROPERTIES AND COMMUNITY STABILITY}

Several food web properties were measured before and after invasion (Figure 1). These included coarse-grained properties: number of species $(S)$, connectance $\left(C=L / S^{2}\right)$, and links per species $(L / S)$, and fine-grained properties, including mean food chain length (MFCL), number of food chains (ChnNo), standard deviation of the generality (GenSD) and vulnerability $(V u l S D)$, fraction of producer $(\% B)$, intermediate $(\% I)$, and top predator $(\% T)$ species, and modularity [the degree to which the food web is modular, calculated using the netcarto algorithm (Guimerà and Amaral, 2005)]. GenSD and VulSD quantify the variability in the standardized number of prey $\left(G_{i}\right)$ and predators $\left(V_{i}\right)$ of the species in the network respectively:

$$
G_{i}=\frac{1}{L / S} \sum_{j=1}^{S} a_{j i} \quad V_{i}=\frac{1}{L / S} \sum_{j=1}^{S} a_{i j}
$$

where $a_{i j}$ is equal to 1 whenever there exists a link between prey $i$ and predator $j$.

Community dynamics were iterated for a period of 2000 time steps before introducing the invasive species (Figure 1). This was enough to reach stable dynamics of total community biomass (see below), in most of our simulations. A species was considered extinct if its biomass fell below $10^{-9}$ (i.e., they stop having any observable effect on community dynamics).

We measured two types of stability: (i) total community biomass stability and (ii) average coefficient of variation $(\mathrm{CV})$ of species biomass. Total community biomass $(T B)$ was measured as the sum of the biomasses of all species in the community at any given moment, and its coefficient of variation was given by

$$
C V_{T B}=\frac{\sigma_{T B}^{2}}{\overline{T B}}
$$

with the mean and SD calculated over 200 time steps. Per species population biomass stability $(P B)$ was measured for each species $i$ over the same 200 time steps, and the average $C V\left(C V_{P B}\right)$ for all species was given by

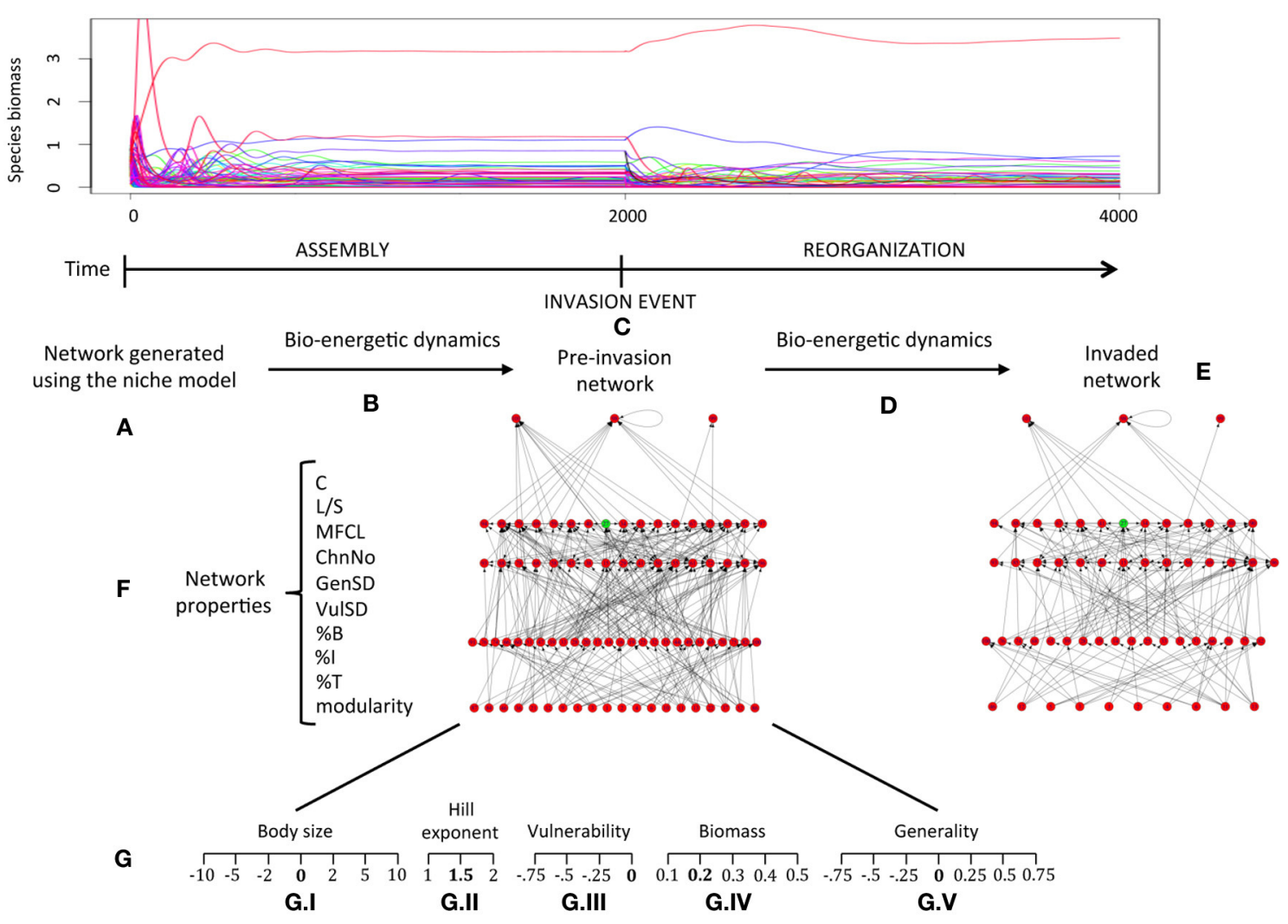

FIGURE 1 | Conceptual diagram of the in silico experiments performed. (A) The initial food web structure of the community is generated using the niche model. (B) Community dynamics through a transient period (from iteration 0 to 2000) are simulated using the bio-energetic model (see text). Communities that are stable at time step 2000 are subject to invasion (C). After invasion, the community evolves further 2000 time steps (to 4000) (D) until it reaches a new state $(\mathbf{E})$. Before introducing the invasive species a series of network properties are measured $(\mathbf{F})$. These properties are again measured in $(\mathbf{E})$ for comparison against their original values in (C) (before invasion). This process is repeated with several values for 5 different species traits (G.I-V) in order to test for the influence of variability within these traits on invasion success (see description of these experiments in the text, including the meaning of each trait value). 


$$
\frac{1}{S} \sum_{i} C V_{i}=\frac{1}{S} \sum_{i} \frac{\sigma_{\mathrm{Ni}}^{2}}{\overline{\mathrm{N}}_{\mathrm{i}}}
$$

Following Loreau and de Mazancourt (2013) and the fact that the variance of a sum of variables is the sum of the variances and covariances of all these variables, we can derive under which circumstances stability in terms of $T B$ is achieved earlier than stability in terms of $P B$, resulting in:

$$
\sum_{\mathrm{i}} \sum_{j} \operatorname{cov}>\overline{T B} x C V_{P B}-\sum_{i} v a r
$$

where cov corresponds to covariances among species pairs $i$ and $j$, var to variances of species $i$.

We assumed a community achieved total biomass stability when $C V_{T B}<0.1$, and species biomass stability when $C V_{P B}<0.1$. The $10 \%$ thresholds were arbitrarily chosen as a way of quantifying the maximum amount of change allowed before becoming unstable.

\section{IN SILICO INVASION EXPERIMENTS}

We ran the niche model for the number of species in the native community plus one (i.e., $S=60+1$ ). The invasive species was then selected randomly from the pool of non-basal species yielded by the niche model and removed from it, together with its interactions. Invasive species were not allowed to be basal species because these cannot go extinct under the current experimental setting, and would bias our results. In this way the original community was formed, along with the invasive species that was going to be introduced into that same community later. By generating the invasive species and its interactions following the same heuristic implemented by the niche model, we ensure an empty niche space that the invader is best placed to occupy. In this manner, the standard invader possesses traits and interactions similar to those from remaining species in the native food web. Then, by switching both traits and interactions, it is possible to systematically explore specific hypothesis outlined above.

In order to ensure that our experiments were all performed under similar initial conditions across the different values of connectance considered and to avoid biases in our results due to differences in initial conditions, the communities over which invasion experiments were performed were subject to a rigorous selection process in terms of species richness and stability. Simulated communities were selected based on whether they (i) possessed at least $80 \%$ of the initial number of species (i.e., 48), and (ii) were stable in terms of total community biomass after reaching time step 2000 (Figure 1). A number of simulations were performed for each of the values of connectance considered $(0.05,0.1,0.15)$ until 300 communities meeting the criteria outlined above (i.e., possessing 48 species or more, and being stable in terms of total community biomass) were obtained for each connectance value, yielding a total of 900 distinct model communities. Mean species richness (s.d.) for the food webs obtained is 56 (2.38), 58 (1.86), and 59 (1.64); while mean final connectance (s.d.) is 0.055 (0.0075), 0.098 (0.0139), and 0.139 (0.0159) for each group of webs with original connectance values of $0.05,0.1$, and 0.15 , respectively. The average number of species in the resulting communities was 58; and there was no a priori correlation between species richness and connectance in this set of communities.

For the selected communities, we measured the food web properties described above, and then performed the introduction of the invasive species. We simulated 2000 additional time steps (for a total of 4000), and computed food web properties again (Figure 1). Invasion success was assessed as the persistence of the invasive species (whether its biomass dropped below $10^{-9}$ ) before reaching the end of the simulation (iteration 4000).

In addition to the "standard" invasion experiment,-i.e., with the default values for all of the species traits (values in bold under each experiment G.I-V in Figure 1) - we performed several additional invasion experiments in order to test for the effects of particular species traits on invasive success and the relative relevance of these traits across communities with different connectivity. Simulations were thus replicated on each of our selected communities by varying the following traits (Figure 1), which allowed us to test our hypotheses:

(1) Body size: where the invasive could be 2, 5, or 10 times bigger or smaller than in the standard experiment.

(2) Predation strategy: where the invasive could take two distinct values for the Hill exponent, 1 and 2, simulating Holling type II and type III functional responses for the invasive consumer respectively.

(3) Generality: where the invasive species could have 25, 50, or $75 \%$ of its incoming links (as defined by the niche model) either removed or added, effectively diminishing or augmenting its prey number respectively. In the case of links removed, these were randomly chosen from the available prey for that species. In the case of added links, these were randomly selected from the pool of species that were prey of consumers from the same trophic level of the invasive.

These 3 sets of experiments allowed us to test specifically the mechanism of prey consumption as a determinant of invasion success.

(4) Vulnerability: where the invasive species could be 25,50 , or $75 \%$ less vulnerable (i.e., have that fraction of reduced predation) than originally defined by the niche model. Predatory links removed were chosen randomly amongst the outgoing links of the invasive species.

This set of experiments allowed us to test the mechanism of predator release as a determinant of invasion success by allowing for a comparison between different levels of predation pressure over the same species.

(5) Abundance: where the initial biomass of the invasive species took values of either $0.1,0.3,0.4$, or 0.5 (default value in standard experiments was 0.2 ). These values were chosen because they fall well within the values of the distribution from which the initial biomasses of species in the communities were taken (see Supplementary Material S2). These were considerably lower that those employed in other studies that have used the same model for population dynamics employed here (Romanuk et al., 2009), in order to account for the fact that invasive species are usually less abundant than those in the 
native community. This allowed us to investigate the effect of propagule pressure on invasion success.

The configurations described above yield a total of 22 distinct invasion experiments for each generated community $(22 \times 900=19,800$ simulations $)$. The model and the statistical analyses were implemented using the $\mathrm{R}$ statistical package ( $\mathrm{R}$. Development Core Team, 2009).

\section{RESULTS \\ IS FOOD WEB COMPLEXITY AND STRUCTURE A GOOD PREDICTOR OF INVASION SUCCESS?}

More complex networks were more vulnerable to invasion. Higher connectivity (both in terms of $C$ and $L / S$ ), and larger MFCL and ChnNo were significantly and positively associated with susceptibility to invasion (Figure 2, Table S3). Similarly, networks with a lower standard deviation of the generality (GenSD), a smaller fraction of basal $(\% B)$ and top predator $(\% T)$ species, and lower modularity were more prompt to invasion (Figure 2, Table S3).

However, as many of these properties are highly correlated with connectance, once we compare food webs with similar connectance values (in our simulations $0.05,0.1$, and 0.15 ), only a few of these properties affect invasion success (Table S4).

Our finding thus suggest that increasing connectivity has an effect on network robustness and an important effect on food web
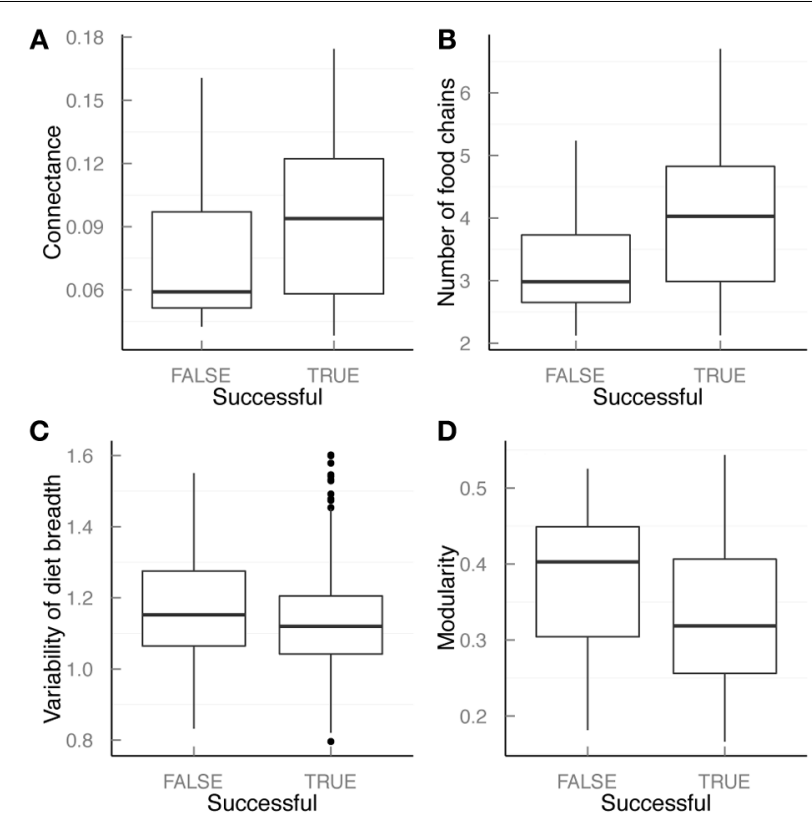

FIGURE 2 | Relationship between food web properties and invasion success. In each panel, box plots correspond to the median value and the first (bottom of the box) and third (top of the box) quartiles of the distribution of each network property: (A) connectance $\left(C=L / S^{2}\right)$, (B) Number of food chains $(\log (\mathrm{ChnNo}))$, (C) Standard deviation of generality (GenSD), and (D) modularity. In each panel, values of each property in communities with successful (True) and unsuccessful (False) invasions are compared. properties that are relevant for the internal organization of the network of interactions.

\section{DO INVASIONS MAKE FOOD WEBS LESS COMPLEX?}

The main effect of invasions was species loss, with communities losing on average $8(S D=6)$ species. Basal species were predominantly loss, decreasing their fraction in the post-invasion network, which in turn increased the fraction of top predators and intermediate species. In general, food web complexity was reduced. While invasions increased connectance, other aspects of complexity were significantly reduced, with networks becoming less modular, and having less food chains (Supplementary Material S3 and Table S5). Similarly, the standard deviation of generality decreased following invasions. This suggests that communities were losing resource input channels, with species composition getting biased toward higher trophic levels, and with consumers becoming more homogeneous in the number of prey they attack (Table S6). Species loss was detrimental for community robustness, because it increased connectance among the remaining species in the community, ultimately favoring further invasions. Invasive species were beneficial for the persistence of more generalist species which in turn resulted in changes in other food web properties (e.g., modularity, heterogeneity of diet breadth).

When invasion attempts failed some of these food web properties also changed. However, the effects caused by successfully established invaders were consistently stronger than those caused by unsuccessful invaders (data not shown).

\section{DO INVADER TRAITS AFFECT THEIR INVASION SUCCESS?}

\section{Are bigger species better able to invade communities?}

Larger sizes of a given species were more successful invading a native community than smaller sizes (Figure 3A, Table 1), everything else being equal. In the model used in this work the effect of species body size affects the community via the metabolic rate of that species, which in turns determines how much resource a given species gathers from its prey. Even though in our case the ultimate determinant of changes in metabolic rates is body size, the former could change due to other factors (e.g., increasing temperature) and the effects of this change would be similar to what is shown here. We can expect then that changes in metabolic rates (regardless of its ultimate cause) will have a strong role on invasion success.

The effects of body size on invasion success varied among communities with different connectance values. We found that it was more important to be bigger when invading less connected communities (Figure 3A, Table S6). This agrees with our finding that food webs with lower connectance were generally harder to invade (Figure 2A). For a given species, being large is always beneficial for their establishment success. Although not conclusively, our results seem to suggest that this happens only up to a certain point, after which, no matter how large the species is, its likelihood of being successful does not increase (Figure 3A). Bigger species (or those increasing their metabolic demands) will be generally more successful invaders although they will find it harder to invade communities that are loosely connected. Under the current global change scenario, communities are probably getting 


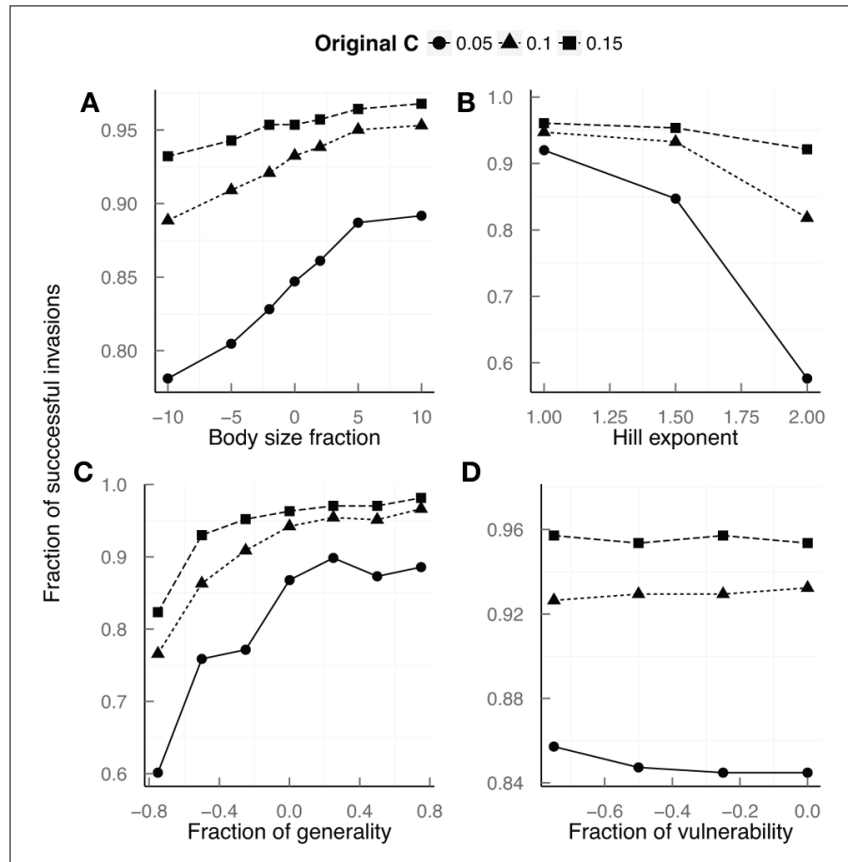

FIGURE 3 | Effects of invader species traits on invasion success measured as the fraction of successful invasions for a given trait value.

(A) Body size: values represent species 10 ( -10 and 10), 5 (-5 and 5), and $2(-2$ and 2 ) times smaller $(-)$ or bigger $(+)$ than the reference value for that species according to the bio-energetic model. (B) Ability of the invasive species to capture prey (Hill exponent). (C) Fraction of generality: fractions represent invasive species with $25 \%(-0.25$ and 0.25$), 50 \%(-0.5$ and 0.5$)$, and $75 \%(-0.75$ and 0.75$)$ less (-) or more $(+)$ prey than the species reference value. (D) Fraction of vulnerability: values represent invasive species with $25 \%(-0.25), 50 \%(-0.5)$, and $75 \%(-0.75)$ less predators than the reference. Circles, triangles, and squares correspond to the values of the fraction of invasion success for networks with connectance values of $0.05,0.1$, and 0.15 , respectively.

simpler due to the loss of interactions, although in some cases the opposite might be true (Lurgi et al., 2012a). If the former case is the norm, we should expect species to be bigger if they are going to thrive in novel communities, as has been shown the case in, for example, mountain ecosystems (Lurgi et al., 2012b). Our results agree with previous findings that suggest that novel communities will be biased toward species with larger body sizes that are easily capable of invading native communities (Lurgi et al., 2012a).

\section{Is predation strategy a good predictor of invasion success?}

As expected, our results suggest that invasive species that are better able to capture prey at low densities are more successful invaders. As for the effects of body size, it is more important to have a better predation strategy in communities with lower values of connectance, which, as shown before, are harder to invade (Figure 3B and Table S7). In this case, however, the difference between the two extremes (i.e., functional response type II vs. functional response type III) is more pronounced than for the body size-connectance relationship, with differences up to $40 \%$ in the fraction of successful invasions for less connected food webs (Figure 3B). Differences between displaying the default functional response (i.e., Hill exponent $=1.5$ ) and
Table 1 | Effects of variation on invader species traits on invasion success.

\begin{tabular}{|c|c|c|c|c|}
\hline Trait name & Trait value & Coefficient & $z$ & $p$-value \\
\hline \multirow[t]{6}{*}{ Body size } & -10 & -0.46285 & -3.330 & $<0.001$ \\
\hline & -5 & -0.29189 & -2.048 & 0.041 \\
\hline & -2 & -0.12847 & -0.877 & 0.3804 \\
\hline & 2 & 0.10537 & 0.688 & 0.4914 \\
\hline & 5 & 0.33213 & 2.064 & 0.039 \\
\hline & 10 & 0.39218 & 2.403 & 0.016 \\
\hline \multirow[t]{2}{*}{ Hill exponent } & 1 & 0.5261 & 3.109 & 0.002 \\
\hline & 2 & -1.2171 & -9.344 & $<0.001$ \\
\hline \multirow[t]{4}{*}{ Biomass } & 0.1 & $-5.992 e-15$ & 0 & 1 \\
\hline & 0.3 & 1.130e-02 & 0.075 & 0.9401 \\
\hline & 0.4 & $1.130 \mathrm{e}-02$ & 0.075 & 0.9401 \\
\hline & 0.5 & $1.130 \mathrm{e}-02$ & 0.075 & 0.9401 \\
\hline \multirow[t]{6}{*}{ Generality } & -0.75 & -1.5640 & -11.303 & $<0.001$ \\
\hline & -0.5 & -0.7896 & -5.372 & $<0.001$ \\
\hline & -0.25 & -0.5716 & -3.787 & $<0.001$ \\
\hline & 0.25 & 0.2767 & 1.570 & 0.1163 \\
\hline & 0.5 & 0.1003 & 0.592 & 0.5538 \\
\hline & 0.75 & 0.3117 & 1.755 & 0.0793 \\
\hline \multirow[t]{3}{*}{ Vulnerability } & -0.75 & $4.670 e-02$ & 0.306 & 0.7599 \\
\hline & -0.5 & $1.410 \mathrm{e}-14$ & 0 & 1 \\
\hline & -0.25 & $3.467 e-14$ & 0 & 1 \\
\hline
\end{tabular}

GLMs results presented correspond to the difference between the trait values on the table and the default value for each trait respectively (see text). Food web connectance was included into the GLM as a fixed factor. Values for each trait are defined in the caption of Figure 3 and Methods. The sign of the coefficient value represents the directionality of the relationship between that trait and the fraction of invasion success. Degrees of freedom for the models measuring the effect of body size $=7321$, hill exponent $=3137$, biomass $=5229$, generality $=6964$, and vulnerability $=4103$.

having a Holling type III functional response made invasive species significantly more successful (Table 1). However, differences between the type II functional response and the default one, although still significant, were not as marked (Table 1). Predation strategy-conceived as the ability of an introduced species to capture prey-is rarely determined in studies of invasion success. Here we found that is an important determinant of invasion success. This highlights the importance of focusing on alternative species traits when assessing the likeliness of invasion. Our results agree with previous theoretical findings where species that were able to gather more resources were in turn more likely to invade communities (Galiana et al., 2014). This points to the need of incorporating attack rates and assimilation efficiencies as important species traits in a community framework of invasions.

\section{Is species biomass an important determinant of invasion success?}

The biomass of the invader species at the time of introduction did not affect its invasion success. For all the values tested in our trials the percentage of successful events relative to the total number of attempts remained constant (Table 1). It is important 


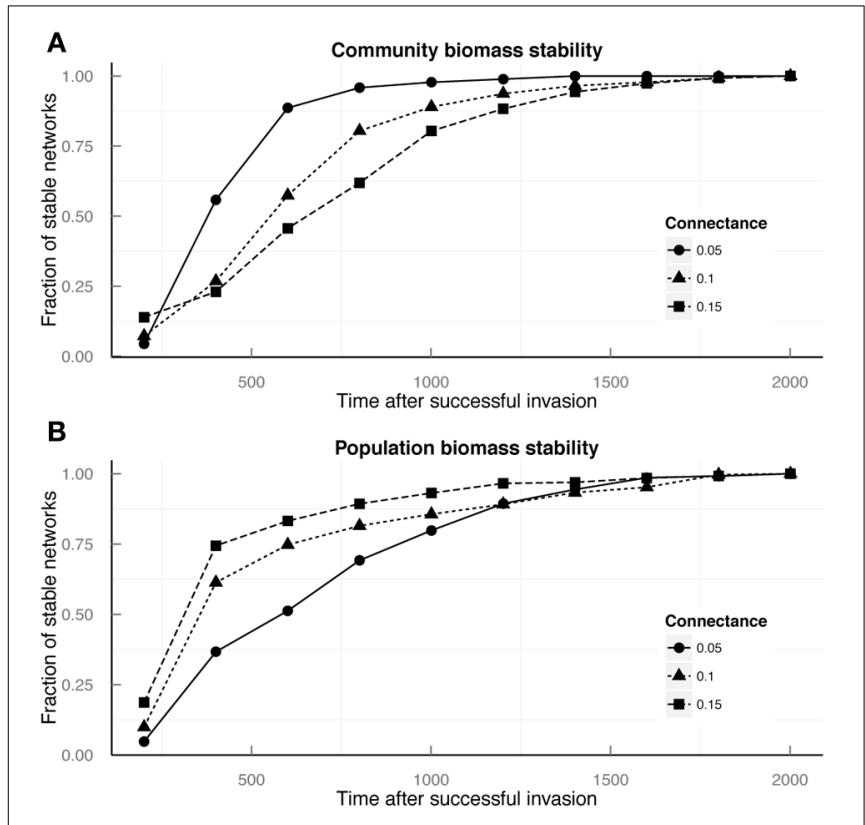

FIGURE 4 | Food web stability analysis for communities with successful invasions. Cumulative fraction of stable networks is presented in terms of $\mathbf{( A )}$ total community biomass, and $(\mathbf{B})$ mean species biomass $\mathrm{CV}$, plotted against the time elapsed since the invasion. Circles, triangles, and squares represent the fraction of communities achieving stability at that time step for communities with connectance values of $0.05,0.1$, and 0.15 respectively.

to note however, that even when we chose values for the initial biomass of the invasive species in the lower part of the range of the biomasses of species in the communities at the commencement of the simulations, native species biomasses dropped significantly after the preinvasion period had lapsed. At the time of the invasive's introduction, 36,40 , and $48 \%$ of the species in communities with connectances of $0.05,0.1$, and 0.15 , respectively, had biomass values less than 0.1 . The mean (median) biomass values in these communities were 0.2335 (0.1554), 0.3654 (0.1541), and 0.4765 (0.1534), respectively. It is nonetheless interesting that even for invaders entering the community with a biomass value of 0.1 , which is smaller than $64 \%$ of the native species in the case of less connected communities, the fraction of successful invasions is comparable to that observed for invaders with much larger biomasses.

\section{Are more generalist species more successful invaders?}

We found that, for species occupying the same position within the food web, when varying the number of prey species they feed on, those attacking a smaller number of different prey items were less successful invaders (Figure 3C). On the other hand, more generalist consumers were better at invading communities only until a given threshold, beyond which increasing the number of different prey species consumed did not affect its probability of invading successfully. The fraction of successful invasions reached a plateau and then remained relatively constant at values of 0.25 of the fraction of generality, i.e., having 25\% more prey items had the same effect on invasion success than having $75 \%$ more
(Figure 3C and Table 1). In agreement with our previous results, it was more important to be more generalist in food webs with lower connectance values that are harder to invade. For example, for an invasive species that is $75 \%$ less generalist than expected by its niche value, the difference between successful and unsuccessful invasion attempts can go up to more than $20 \%$ between food webs with $C=0.05$ against networks with $C=0.15$ (Figure 3C and Table S8).

\section{Is vulnerability a good predictor of invasion success?}

Changes in the number of predators attacking the invasive species did not affect its probability of success (Figure 3D). Even for large fractions of removed predatory links (e.g., -0.75), the only noticeable increase in invasion success was observed for poorly-connected webs, with the fraction of successful invasions increasing by only $1 \%$ in comparison to the case in which the invader kept all of its predatory links given by its niche position (Table 1). Again, less connected webs were harder to invade, but predator release was not a strong determinant of invasion success. Although traditionally considered an important determinant of invasion success (e.g., Keane and Crawley, 2002; Colautti et al., 2004), we did not find that enemy release was an important predictor for invasion success. Even in communities that are harder to invade, other species traits might be more important for establishment than a release from detrimental biotic interactions.

\section{COMMUNITY STABILITY}

We found that network connectance determined which of the 2 stability measures (i.e., total community biomass, $T B$, or population biomass stability, $P B$ ) was achieved earlier after the invasion. In poorly connected food webs $(C=0.05), 41 \%$ of the times $T B$ stability was achieved earlier than $P B$ stability, while only in $25 \%$ it was the other way around. In contrast, highly connected food webs $(C=0.15)$, after the invasion, tend to recover faster $P B$ (63\% of the times) than $T B$ (18\% of the times) stability. Food webs with intermediate connectance $(C=0.10)$ behave like highly connected ones, although differences in achieving earlier each type of stability were less marked (Figure 4).

Equation (5) gives the conditions under which $T B$ stability is achieved earlier than $P B$ stability, predicting that species covariances need to be more common and stronger than in those communities where $P B$ stability is achieved earlier. We observed this trend in communities achieving $T B$ stability earlier than $P B$ stability among our model communities after the introduction of the invasive species. In particular, we found the most significant covariances were negative and among basal species and between basal species and herbivores (Supplementary Material S4). As mentioned above, less connected communities, which generally achieve $T B$ stability faster than $P B$ stability, are more resistant to invasions. These facts taken together suggest that, both indirect competition between basal species and predator-prey dynamics at the bottom of the food web might be a mechanism behind the robustness of complex ecological communities to biological invasions. Communities that maintain a relatively constant total biomass in spite of fluctuations at the species' population level (i.e., achieving $T B$ stability earlier than $T B$ stability) may be more resistant to species invasions disrupting population dynamics. 


\section{DISCUSSION \\ COMPLEXITY AND ROBUSTNESS TO INVASIONS}

The link between community complexity and response to disturbances (i.e., stability and robustness) has been the subject of a long, heated, and far from finished debate in ecology (Pimm, 1991; McCann, 2000; Montoya et al., 2006). In the face of current global change, this research area has been reinvigorated. Here we have shown how food web structure and complexity mediates the vulnerability of communities to biological invasions. We found that more connected food webs are more vulnerable to invasions. This contrast with previous theoretical results by Romanuk et al. (2009), where the authors found that more connected food webs were more robust to invasions. These opposite results may arise from the relationship between species richness and connectance in both studies. Romanuk et al. used less speciose food webs than the ones used here ( 15 species on average in comparison to the 58 species used in the present study), but more importantly, in that study, more connected food webs were systematically less diverse. Theoretical and empirical studies generally show that higher levels of diversity confer resistance to invasions (e.g., Case, 1990; Pimm, 1991; Kennedy et al., 2002), and thus the effects of richness and connectance can be confounded. In this work we isolated the effect of connectance on invasion success by keeping diversity constant and relatively large-all the food webs selected for the experimental invasions had 48 species or more, regardless of connectance. Once assessed independently and in relatively large food webs, higher levels of connectance likely increase vulnerability to invasions.

We found that not only complexity, but also the internal organization of links within the food web, as measured by, e.g., modularity or diet breadth variability, affected the vulnerability of communities to invasions. However, these effects were indirect and highly mediated by connectance, as most food web properties are a consequence of varying connectance values (Riede et al., 2010). Food webs containing consumers with very diverse diet breadths (from very specialized to generalists) were more robust to invasions. These communities limit the chances of success of an invader consuming a sufficient amount of prey, since most prey are already exploited by different specialized and generalist consumers. Communities with higher connectance necessarily bias the diet breadth continuum toward more generalist consumers. This could in turn allow for specialized consumers to be able to thrive by gathering enough resources from only a handful of prey and sharing its predators with many others.

Food web modularity has been shown to be a relevant feature for community stability and robustness. It has been shown that modular architectures prevent the propagation of perturbations affecting population abundances across the whole food web (Montoya and Solé, 2002; Stouffer and Bascompte, 2011). Our results extend these previous findings to the context of biological invasions. In our model communities, those weakly connected and more modular were the most robust against invasions. Coincidentally, Thébault and Fontaine (2010) found that precisely these are the features that confer stability to food webs. Stability thus, given by high modularity and low connectance, is a possible mechanism behind robustness to invasion in complex model communities.
We observe a paradoxical relationship between complexity and stability after invasions. Less connected food webs showed a higher stability in terms of total community biomass but lower stability in terms of variation on individual species biomass. In these communities, we identified a larger number of negative covariances between species populations both within the same trophic level (for basal species) and across trophic levels. This mirrors recent experimental and theoretical work on the relationship between diversity and stability in competitive communities (Tilman et al., 2006; Hector et al., 2010; Loreau and de Mazancourt, 2013). In those studies diversity was found to stabilize aggregate community or ecosystem properties while simultaneously destabilizing individual species abundances. Our results complement these studies by suggesting that lower connectance but highly coupled population dynamics through negative covariances (both within and across trophic levels) result in larger ecosystem- than population-stability and in communities more resistant to invasions. Communities that are more stable in terms of total biomass fluctuations, even when allowing for fluctuations at the species level, are thus more robust against biological invasions.

\section{THE EFFECTS OF INVASIONS OVER COMPLEX COMMUNITIES}

Although widely recognized to have detrimental effects on ecosystem functioning and population abundances (e.g., Simberloff et al., 2013), the effect of invasions on community structure and organization has been less explored (but see Dijkstra et al., 2013; Salvaterra et al., 2013).

One of the main consequences of invasions in our complex model food webs was biodiversity loss, which in turn prompted important changes in food web structure. These network-level changes might be linked to those caused by the cascade of biodiversity loss that follows primary extinctions linked to the invasion event. Our post-invasion model communities became more connected, less modular, more homogeneous in terms of diet breadth, and with a smaller fraction of basal species. These novel features are similar to those that we identified as characteristic of communities that are more susceptible to invasions, showing that biodiversity loss makes communities more vulnerable to further perturbations (Tilman, 1999; Borrvall et al., 2000).

A homogenization in terms of diet breadth and a decrease in the fraction of basal species affect the number of energy input channels in the food web. Fewer basal species are available for species in higher trophic positions, and most of the latter are generalists (since post-invasion communities are also more connected). This decreases the energy available and generates more competition for existing resources. Novel communities resulting from invasions are not only becoming less diverse in terms of species and interactions but are potentially resulting in higher levels of competition among the remaining species.

\section{BIOLOGICAL INVASIONS AND SPECIES TRAITS}

The identification of key species traits that predict invasion success is pivotal in invasion ecology (e.g., Cassey et al., 2004; Jeschke and Strayer, 2006). Here, we have tested the effects of key species traits, some of which have traditionally been studied 
in the literature of invasions ecology, on invasion success. This allowed us to test ecological mechanisms like "enemy release," or propagule pressure that have been proposed as important determinants of invasion success (e.g., Elton, 1958; Keane and Crawley, 2002; Blackburn et al., 2009). Traditionally considered an important mechanism behind invasion success, the enemy release hypothesis has recently been re-analyzed and shown not be as determinant as previously thought, its effects being confounded by other factors (Colautti et al., 2004). Here we have shown that, at least in theoretical models, predator release does not seem to be a relevant mechanism for the success of invasive species. This highlights the importance of a more comprehensive view of biological invasions in complex food webs, pointing to the plausibility of the effects of enemy release, and possibly other mechanisms too, being confounded by other factors in empirical observations.

Similarly, our results suggest that propagule pressure (considered here simply as the biomass at the time of the introduction), an important predictor of invasion success in the literature (Jeschke and Strayer, 2006), had a negligible effect in our theoretical approximation. This might be due to our choice of biomass ranges at the time of introduction, as we used biomasses within the range of those of the species present in the community, whereas in most natural settings the biomass (or abundance) of introduced species are well below the numbers of many native species. It might be noted however, that in empirical studies, propagule pressure might be an important predictor of invasion success mostly due to stochastic extinctions of invaders at low abundances, which are not contemplated in our model. Our theoretical results thus suggest that if the introduced species has a population size above some threshold (e.g., similar to native species), then the exact number of individuals introduced is no longer a strong determinant. Propagule pressure however has been linked to resource availability (Davis et al., 2000), suggesting that this will be a strong mechanism preventing invasions only to the extent that it is at play in tandem with a low availability of resources. In our communities resources did not fluctuate at the time of introduction since we explicitly selected stable communities to perform our invasion experiments on. This suggests that perhaps in nature, propagule pressure must be accompanied by other factors such as resource fluctuations in order to have a strong controlling effect over invasions.

Two species traits that did positively influence the success of establishment of invasive species in our model communities were body size and predation strategy, in agreement with recent empirical observations (e.g., Blackburn et al., 2009). Recent theoretical work on invasions on mountain ecosystems (Galiana et al., 2014), showed that species from high trophic levels, commonly bigger than those from lower trophic levels, interacting with numerous prey, and having a strong effect on them (which is related to the functional response of a predator) are important determinants of invasion success. Our findings support these observations and the prediction that we can expect novel communities to be less speciose, more connected, and biased toward species with larger sizes.

\section{LIMITATIONS AND STRENGTHS OF THE APPROACH}

The framework presented here has some caveats but also presents some advantages over existing approaches to biological invasions. Species introduced to our model communities are drawn from the same niche model that was employed to generate them. This could be considered a bias toward a particular type of species that can be introduced. However, it can also be seen as a good way to vary species traits in a systematic manner and compare the effects of introducing species belonging and not belonging to the niche of the native species. Admittedly, other types of invasive species could be considered in order to make our study even more comprehensive.

In this study the biomass of the invasive species at the time of introduction took values close to those of an important fraction of species already present in the community. In this sense we obtained high values of invasion success, but this allowed us to discern which network structures and species traits are responsible for differences in invasion success when species are introduced at biomass values of the order of already established species.

Overall, the framework presented in this paper remains a comprehensive way of looking at biological invasions from a complex community perspective, considering the whole picture of interactions the invasive species are embedded in. It can also be extended to test other traditional hypotheses in the realm of biological invasions such as community meltdown (Simberloff and Von Holle, 1999), or whether invasive species are drivers or passengers (MacDougall and Turkington, 2005). This will contribute toward a broader understanding of biological invasions within the context of the complex ecological communities where they occur.

\section{ACKNOWLEDGMENTS}

We thank the reviewers for helpful comments that greatly improved this manuscript. Miguel Lurgi is supported by Microsoft Research, through its PhD Scholarship Programme. José M. Montoya is supported by grants RYC-892 2008-03664, CGL2010-20091, and 2009SGR142.

\section{SUPPLEMENTARY MATERIAL}

The Supplementary Material for this article can be found online at: http://www.frontiersin.org/journal/10.3389/fevo.2014. 00036/abstract

\section{REFERENCES}

Binzer, A., Brose, U., Curtsdotter, A., Eklöf, A., Rall, B. C., Riede, J. O., et al. (2011). The susceptibility of species to extinctions in model communities. Basic Appl. Ecol. 12, 590-599. doi: 10.1016/j.baae.2011.09.002

Blackburn, T. M., Cassey, P., and Lockwood, J. L. (2009). The role of species traits in the establishment success of exotic birds. Glob. Change Biol. 15, 2852-2860. doi: 10.1111/j.1365-2486.2008.01841.x

Blackburn, T. M., Pyšek, P., Bacher, S., Carlton, J. T., Duncan, R. P., Jarošík, V., et al. (2011). A proposed unified framework for biological invasions. Trends Ecol. Evol. 26, 333-339. doi: 10.1016/j.tree.2011.03.023

Borrvall, C., Ebenman, B., and Jonsson, T. (2000). Biodiversity lessens the risk of cascading extinction in model food webs. Ecol. Lett. 3, 131-136. doi: 10.1046/j.1461-0248.2000.00130.x

Bright, C. (1998). Life Out of Bounds: Bioinvasion in a Borderless World. New York, NY: W.W. Norton and Company. 
Brose, U., Williams, R. J., and Martinez, N. D. (2006). Allometric scaling enhances stability in complex food webs. Ecol. Lett. 9, 1228-1236. doi: 10.1111/j.14610248.2006.00978.x

Cardinale, B. J., Srivastava, D. S., Duffy, J. E., Wright, J. P., Downing, A. L., Sankaran, M., et al. (2006). Effects of biodiversity on the functioning of trophic groups and ecosystems. Nature 443, 989-992. doi: 10.1038/nature05202

Case, T. J. (1990). Invasion resistance arises in strongly interacting species-rich model competition communities. Proc. Natl. Acad. Sci. U.S.A. 87, 9610-9614. doi: 10.1073/pnas.87.24.9610

Cassey, P., Blackburn, T. M., Sol, D., Duncan, R. P., and Lockwood, J. L. (2004). Global patterns of introduction effort and establishment success in birds. Proc.R. Soc. B Biol. Sci. 271, 405-408. doi: 10.1098/rsbl.2004.0199

Colautti, R. I., Ricciardi, A., Grigorovich, I. A., and MacIsaac, H. J. (2004). Is invasion success explained by the enemy release hypothesis? Ecol. Lett. 7, 721-733. doi: 10.1111/j.1461-0248.2004.00616.x

Davis, M. A., Grime, J. P., and Thompson, K. (2000). Fluctuating resources in plant communities: a general theory of invasibility. J. Ecol. 88, 528-534. doi: 10.1046/j.1365-2745.2000.00473.x

Dijkstra, J. A., Lambert, W. J., and Harris, L. G. (2013). Introduced species provide a novel temporal resource that facilitates native predator population growth. Biol. Invasions, 15, 911-919. doi: 10.1007/s10530-012-0339-1

Dunne, J., Williams, R. J., and Martinez, N. D. (2002). Network structure and biodiversity loss in food webs: robustness increases with connectance. Ecol. Lett. 5, 558-567. doi: 10.1046/j.1461-0248.2002.00354.x

Dzialowski, A. R., Lennon, J. T., and Smith, V. H. (2006). Food web structure provides biotic resistance against plankton invasion attempts. Biol. Invasions 9, 257-267. doi: 10.1007/s10530-006-9030-8

Elton, C. (1958). The Ecology of Invasions by Animals and Plants. London, MA: Methuen.

Galiana, N., Lurgi, M., Montoya, J. M., and López, B. C. (2014). Invasions cause biodiversity loss and community simplification in vertebrate food webs. Oikos 123, 721-728. doi: 10.1111/j.1600-0706.2013.00859.x

Guimerà, R., and Amaral, L. A. N. (2005). Functional cartography of complex metabolic networks. Nature 433, 895-900. doi: 10.1038/nature03288

Hector, A., Hautier, Y., Saner, P., Wacker, L., Bagchi, R., Joshi, J., et al. (2010). General stabilizing effects of plant diversity on grassland productivity through population asynchrony and overyielding. Ecology, 91, 2213-2220. doi: 10.1890/09-1162.1

Jeschke, J. M., and Strayer, D. L. (2006). Determinants of vertebrate invasion success in Europe and North America. Glo. Change Biol. 12, 1608-1619. doi: 10.1111/j.1365-2486.2006.01213.x

Keane, R. M., and Crawley, M. J. (2002). Exotic plant invasions and the enemy release hypothesis. Trends Ecol. Evol. 17, 164-170. doi: 10.1016/S01695347(02)02499-0

Kenis, M., Auger-Rozenberg, M.-A., Roques, A., Timms, L., Péré, C., Cock, M. J. W., et al. (2008). Ecological effects of invasive alien insects. Biol. Invasions 11, 21-45. doi: 10.1007/s10530-008-9318-y

Kennedy, T., Naeem, S., Howe, K. M., Knops, J. M. H., Tilman, D., and Reich, P. (2002). Biodiversity as a barrier to ecological invasion. Nature 417, 636-638. doi: $10.1038 /$ nature 00776

Koen-Alonso, M. (2007). "A process-oriented approach to the multispecies functional response," in From Energetics to Ecosystems: The Dynamics and Structures of Ecological Systems. The Peter Yodzis Fundamental Ecology Series, eds N. Rooney, K. S. McCann and D. L. G. Noakes (Dordrecht: Springer), 1-36.

Kolar, C. S., and Lodge, D. M. (2002). Ecological predictions and risk assessment for alien fishes in North America. Science 298, 1233-1236. doi: 10.1126/science. 1075753

Loreau, M., and de Mazancourt, C. (2013). Biodiversity and ecosystem stability: a synthesis of underlaying mechanisms. Ecol. Lett. 16, 106-115. doi: 10.1111/ele.12073

Lurgi, M., Lopez, B. C., and Montoya, J. M. (2012a). Novel communities from climate change. Philos.Trans. R. Soc. B Biol. Sci. 367, 2913-2922. doi: 10.1098/rstb.2012.0238

Lurgi, M., Lopez, B. C., and Montoya, J. M. (2012b). Climate change impacts on body size and food web structure on mountain ecosystems. Philos. Trans. R. Soc. B Biol. Sci. 367, 3050-3057. doi: 10.1098/rstb.2012.0239

MacDougall, A. S., and Turkington, R. (2005). Are invasive species the drivers or passengers of change in degraded ecosystems? Ecology 86, 42-55. doi: $10.1890 / 04-0669$
McCann, K. S. (2000). The diversity-stability debate. Nature 405, 228-233. doi: $10.1038 / 35012234$

Montoya, J. M., Pimm, S. L., and Solé, R. V. (2006). Ecological networks and their fragility. Nature 442, 259-264. doi: 10.1038/nature04927

Montoya, J. M., and Solé, R. V. (2002). Small world patterns in food webs. J. Theor Biol. 214, 405-412. doi: 10.1006/jtbi.2001.2460

Montoya, J. M., and Solé, R. V. (2003). Topological properties of food webs: from real data to community assembly models. Oikos 102, 614-622. doi: 10.1034/j.1600-0706.2003.12031.x

Pimm, S. L. (1991). The Balance of Nature? Ecological Issues in the Conservation of Species and Communities. Chicago, IL: University of Chicago Press.

R. Development Core Team. (2009). R: A Language and Environment for Statistical Computing. Vienna: R Foundation for Statistical Computing.

Ricciardi, A., Hoopes, M. F., Marchetti, M. P., and Lockwood, J. L. (2013). Progress toward understanding the ecological impacts of nonnative species. Ecol. Monographs, 83, 263-282. doi: 10.1890/13-0183.1

Riede, J. O., Rall, B. C., Banasek-Richter, C., Navarrete, S. A., Wieters, E. A., Emmerson, M. C., et al. (2010). Scaling of food web properties with diversity and complexity across ecosystems. Adv. Ecol. Res. 42, 139-170. doi: 10.1016/B978-0-12-381363-3.00003-4

Romanuk, T. N., Zhou, Y., Brose, U., Berlow, E. L., Williams, R. J., and Martinez, N. D. (2009). Predicting invasion success in complex ecological networks. Philos. Trans. R. Soc. Lond. B Biol. Sci. 364, 1743-1754. doi: 10.1098/rstb. 2008.0286

Salvaterra, T., Green, D. S., Crowe, T. P., and O'Gorman, E. J. (2013). Impacts of the invasive alga Sargassum muticum on ecosystem functioning and food web structure. Biol. Invasions 15, 2563-2576. doi: 10.1007/s10530-013-0473-4

Shea, K., and Chesson, P. (2002). Community ecology theory as a framework for biological invasions. Trends Ecol. Evol. 17, 170-176. doi: 10.1016/S01695347(02)02495-3

Simberloff, D., Martin, J.-L., Genovesi, P., Maris, V., Wardle, D. A., Aronson, J., et al. (2013). Impacts of biological invasions: what's what and the way forward. Trends Ecol. Evol. 28, 58-66. doi: 10.1016/j.tree.2012.07.013

Simberloff, D., and Von Holle, B. (1999). Positive interactions of nonindigenous species: invasional meltdown? Biol. Invasions 1, 21-32. doi: 10.1023/A:1010086329619

Stouffer, D. B., and Bascompte, J. (2011). Compartmentalization increases food-web persistence. Proc. Natl. Acad. Sci. U.S.A. 108, 3648-3652 . doi: 10.1073/pnas.1014353108

Thébault, E., and Fontaine, C. (2010). Stability of ecological communities and the architecture of mutualistic and trophic networks. Science 329, 853-856. doi: $10.1126 /$ science. 1188321

Tilman, D. (1999). The ecological consequences of changes in biodiversity: a search for general principles. Ecology 80, 1455-1474. doi: 10.2307/176540

Tilman, D., Reich, P. B., and Knops, J. M. H. (2006). Biodiversity and ecosystem stability in a decade-long grassland experiment. Nature 441, 629-632. doi: 10.1038/nature04742

van Kleunen, M., Dawson, W., Schlaepfer, D., Jeschke, J. M., and Fischer, M. (2010). Are invaders different? A conceptual framework of comparative approaches for assessing determinants of invasiveness. Ecol. Lett. 13, 947-958. doi: 10.1111/j.1461-0248.2010.01503.x

Vilà, M., Espinar, J. L., Hejda, M., Hulme, P. E., Jarošík, V., Maron, J. L., et al. (2011). Ecological impacts of invasive alien plants: a meta-analysis of their effects on species, communities and ecosystems. Ecol. Lett. 14, 702-708. doi: 10.1111/j.1461-0248.2011.01628.x

Vitousek, P. M., D’Antonio, C. M., Loope, L. L., and Westbrooks, R. (1996). Biological invasions as a global environmental change. Am. Sci. 84, 468-478.

Wardle, D. A., Bardgett, R. D., Callaway, R. M., and Van der Putten, W. H. (2011). Terrestrial ecosystem responses to species gains and losses. Science 332, 1273-1277. doi: 10.1126/science.1197479

Williams, R. J., and Martinez, N. D. (2000). Simple rules yield complex food webs. Nature 404, 180-183. doi: 10.1038/35004572

Williams, R. J., and Martinez, N. D. (2004). Stabilization of chaotic and non-permanent food-web dynamics. Eur. Phys. J. B 38, 297-303. doi: 10.1140/epjb/e2004-00122-1

Williamson, M. H., and Fitter, A. (1996). The characters of successful invaders. Biol. Conserv. 78, 163-170. doi: 10.1016/0006-3207(96)00025-0

Yodzis, P., and Innes, S. (1992). Body size and consumer-resource dynamics. Am. Nat. 139, 1151-1175. doi: 10.1086/285380 
Conflict of Interest Statement: The authors declare that the research was conducted in the absence of any commercial or financial relationships that could be construed as a potential conflict of interest.

Received: 04 April 2014; accepted: 23 June 2014; published online: 14 July 2014.

Citation: Lurgi M, Galiana N, López BC, Joppa LN and Montoya JM (2014) Network complexity and species traits mediate the effects of biological invasions on dynamic food webs. Front. Ecol. Evol. 2:36. doi: 10.3389/fevo.2014.00036
This article was submitted to Population Dynamics, a section of the journal Frontiers in Ecology and Evolution.

Copyright (c) 2014 Lurgi, Galiana, López, Joppa and Montoya. This is an openaccess article distributed under the terms of the Creative Commons Attribution License (CC BY). The use, distribution or reproduction in other forums is permitted, provided the original author(s) or licensor are credited and that the original publication in this journal is cited, in accordance with accepted academic practice. No use, distribution or reproduction is permitted which does not comply with these terms. 